\title{
Modeling and Simulation Using Cellular Automata
}

\author{
Oliver Ullrich*, Daniel Lückerath
}

Fraunhofer Institute for Intelligent Analysis and Information Systems, Schloss Birlinghoven, 53757 Sankt Augustin, Germany *oliver.ullrich@iais.fraunhofer.de

SNE 30(3), 2020, 125-130, DOI: 10.11128/sne.30.en.10526 Received: July 1, 2020; Revised: August 18, 2020;

Accepted: August 20, 2020

SNE - Simulation Notes Europe, ARGESIM Publisher Vienna, ISSN Print 2305-9974, Online 2306-0271, www.sne-journal.org

Abstract. Cellular Automata are a simple, yet powerful modeling and simulation technique, easily employed if the system to be examined consists of a set of uniform entities that are located in a neighborhood relation to each other. The simple structure and fast execution of Cellular Automata allow for very large models, their inherent parallelism enables a comparatively simple parallelization.

This paper gives an overview on the concepts of Cellular Automata-based modeling and simulation, with a special focus on neighhorhood concepts, border types, and transition functions, and describes current applications of the paradigm in the areas of the simulation of transportation networks, additive manufacturing, as well as urban growth and development. It is aimed at students of the craft as well as practitioners who might want to take a look beyond the GUI of their modeling tools.

\section{Introduction}

Beyond their application in simulation modelling, Cellular Automata (CA) are also studied in other areas of computer science, in mathematics, biology, and physics. CA generally consist of a regular grid of homogenous cells, each of which is assigned one of a finite set of states. A cell changes its state over time based on a transition function - which in turn is determined by the states of a set of other cells, the original cell's neighborhood.

The core concepts of CA were developed in the 1960s by John von Neumann (see [27]) as a tool to formally model self-reproducing biological systems (see [24]). In the 1970s they were developed further, with the aim to examine what was then called "artificial life"
- the best known artifact of that period probably is Conway's Game of Life (see [6]). It was shown that CA are capable of self-reproduction in a way that is sufficient to support a universal computer - they are thus Turing complete (see [24]).

While CA today are applied in a number of domains, for example fault-tolerant quantum computing (see [25]), image encryption (see [19]), modeling of epidemic spreading (see [17]), and object detection in computer vision (see [21]), this paper takes a closer look at their application in the area of modeling and simulation.

In many application fields in the simulation area, CA have been superseded by agent-based modeling and simulation. However, some advantages remain: their simple structure and extremely fast execution allow for very large models, their inherent parallelism enables a comparatively simple parallelization. CA can also be used for high-performance simulation of environments for agent-based models.

This paper gives an introduction on the use of CA in modeling and simulation; it explains both the fundamentals of the technique as well as its applications during the last few years.

The paper continues with a short recap of the basics of simulation modeling (see Section 1), followed by an introduction of CA with a focus on neighborhood concepts and transition functions (see Section 2). It then reports on current applications in modeling and simulation, specifically in the areas of transportation networks, additive manufacturing processes, and urban development simulation (see Section 3). The paper concludes with a short summary of the lessons learned (see Section 4).

\section{Simulation Modeling}

The term simulation can cover a wide variety of processes and models. In its broadest definition, simulation simply refers to the performance of experiments 
on a model in order to make statements about the behaviour of a real system or process (see [16]). A narrower definition of simulation, which is slightly adapted and also used for this work, is formulated by Ingalls (see [8]): Simulation is the performance of experiments on a dynamic and stochastic model with the aim of making statements about the behaviour of a real dynamic and stochastic system or process. Dynamic in this context means that the behavior of the system - and thus of the model - changes over time, while stochastic means that the behavior can be influenced by random variables.

This allows simulation models to be distinguished from (mathematical) analytical models, such as optimization models. Analytical models try to formulate correlations of real systems as sets of mathematical equations. This formulation usually requires the definition of rigid specifications, for example fixed travel times between stops in transit models, in order to obtain manageable models. If, however, the system under consideration meets the requirements, the application of the analytical model always leads to a valid solution. Simulation models, on the other hand, attempt to model the behaviour of the real system as complex behavioral and decision-making processes. This may no longer guarantee that the solution obtained represents an optimum, but allows to represent dynamic and stochastic behaviour (see [12]).

The group of dynamic and stochastic systems includes, for example, technical systems and transportation systems - simulation models are indispensable research tools in those areas. Typically, discrete simulation methods are used to represent such systems, for example CA-based, event-based (see [26]), or agentbased (see [15]) simulation. These paradigms differ in the specific way they represent system components and their interdependencies, as well as in the specific representation of concepts such as time progress. In any case the modeler has to decide which system components and interrelations are critical for representing the behaviour of interest, and which modeling methods are most appropriate (see [12]).

\section{Cellular Automata}

If the system to be simulated consists of a set of uniform entities that are located in a (geographical) neighborhood relation to each other, Cellular Automata can be employed easily and efficiently.
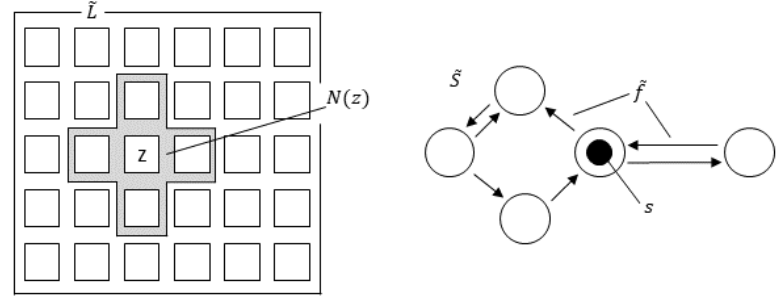

Figure 1: Cellular Automaton $Z=(\tilde{L}, \tilde{S}, N, \tilde{f})$ consisting of a regular, two-dimensional grid $\tilde{L}$ of square-shaped cells $z \in \tilde{L}$ with state $s$ from the set of states $\tilde{S}$ and with a transition function $\tilde{f}$ that is dependent on a neighborhood $N(z)$.

\subsection{Introduction}

A Cellular Automaton $Z=(\tilde{L}, \tilde{S}, N, \tilde{f})$ is a (infinite) regular grid $\tilde{L}$ of cells $z \in \tilde{L}$, each of which may be in any state $s$ from the finite set of states $\tilde{S}$ (see Figure 1). The state of a cell changes over time based on a transition function $\tilde{f}$. Each cell $z$ forms a neighborhood with a finite set of other cells $N(z)=\{i \in \tilde{L} \mid z-i \in N\}$. The characteristics of that neighborhood are determined by the set of permissible neighborhood indices $N$. This set of indices in turn depends primarily on the spatial discretization of the model. In principle, such a lattice geometry can have any number of dimensions; typically, in practical applications one, two, or three dimensional lattices are used. The lattice geometry also defines the shape of the cells. Any cell shape that guarantees the regularity of the grid is conceivable; frequently encountered cell shapes are: square, triangular, or hexagonal. For a more detailed description of these concepts see [28].

\subsection{Neighborhood Concepts}

Figure 2 shows the frequently encountered von Neumann and Moore neighborhood concepts in the context of a number of lattice geometries. As can be seen, the cells of a von Neumann neighborhood of a cell $z$ share a whole edge of the lattice with $z$. The Moore neighborhood, on the other hand, also includes all cells that have only one corner point in common with $z$.

Depending on the characteristics of the system to be modeled, the neighborhood of a cell may not necessarily include only direct neighbors - i.e. cells with distance 1 - but may also include cells further away. That might be useful, if obstacles further away, for example 
Von Neumann
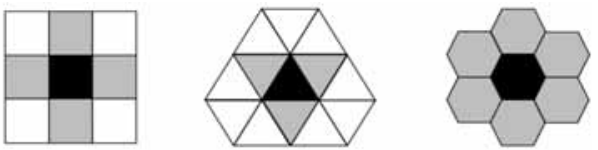

Moore
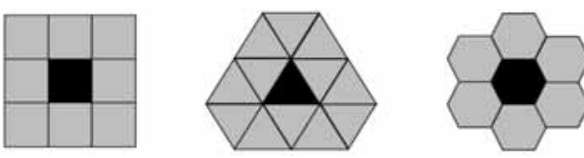

Figure 2: Two often used neighborhood concepts: Von Neumann neighborhoods with shared edges and Moore neighborhoods with shared edges and corner points.

upcoming traffic signals in a public transit simulation, have to be taken into account.

In some models, it is beneficial to define distinct input and output neighborhoods: a cell takes its input from its input neighborhood, while making its state available to the cells of its output neighborhood (see [24]).

For practical applications, the maximum expansion of the grid is usually limited, which makes a specific consideration of border cells necessary. Usually, three different types of border cells are distinguished:

- With periodic borders, opposite sides of the grid are adjacent;

- In case of reflecting borders the border cells reflect the state of the inner cell opposite them; and

- With fixed state borders, the border cells are assigned a fixed state each, which does not change over the course of the simulation.

The grid boundary does not necessarily have to consist of a single border type, but can combine different types.

\subsection{Transition Functions}

When modeling the lattice geometry, decisions have to be made regarding the time progress and the transition function of the CA. In CA the simulation time progresses in fixed increments $\delta t$ between discrete points in time. At each simulation time $t$ the configuration $\tilde{C}_{t}: \tilde{L} \rightarrow \tilde{S}$ assigns a current state to each cell of the grid. The configuration $\tilde{C}_{0}$ designates the start configuration of the CA specified by the modeler. To update the model state over simulation time, a transition function $\tilde{f}: S^{[N \mid} \rightarrow \tilde{S}$ transfers the configuration $\tilde{C}_{t}$ into the configuration $\tilde{C}_{t+1}$ as shown in Equation 1.

$$
\widetilde{C}_{t+1}(z)=\widetilde{f}\left(\left\{\widetilde{C}_{t}(i) \mid i \in N(z)\right\}\right) \quad \forall z \in \widetilde{L}
$$

The transition function is the model characteristic that pivotally determines the behavior of the CA. It depends on the lattice geometry, the type and size of the neighborhood, as well as the number of possible states; its specific design has to be determined together with these model components based on the objectives of the investigation.

Generally, two types of transition functions exist: In a deterministic transition function, state transitions are directly determined by the state of the affected cell and the states of its neighbors. With probabilistic transition functions, the state transition to be applied in each simulation time step is randomly selected from a set of potential state transitions.

Due to the close relationship between lattice geometry, neighborhood, and transition function, spatial and temporal discretization are directly related in CA. Specifically, a decision on the cell dimensions has a direct influence on the size of the time increment $\delta t$. If, for example, a cell length of 30 meters - about one rail car length - is selected for the simulation of light rail systems, a vehicle entity can only move by multiples of these 30 meters within one simulation step. The duration of $\delta t$ has to be assigned accordingly. As a result of these considerations, the duration of one simulation step might be set to correspond to four seconds in the real system (see [10] and [14]), so that vehicles moving at velocities of $0,27,54$, and 81 kilometers per hour can be represented.

The tight interdependencies beween spatial and temporal dimensions do not arise with event-based and agent-based simulation methods, and are one main disadvantage of CA models.

\section{Applications}

CA-based modeling and simulation is utilized in a large variety of subjects. In the following, three areas are examined: the simulation of transportation networks, where CA-based explanatory and predictive models are employed since the 1990s; additive manufacturing, where simulation models help to understand how metal 
powders are melted by a focused laser beam; and urban growth, where simulation studies help to predict how metropolitan regions might develop and how climate change-related effects will impact them.

\subsection{Simulation of Transportation Networks}

One-dimensional CA seem to be a natural choice to model linear transportation infrastructure, for example roads or railways. The CA-based examination of transportation phenomena started out with Nagel and Schreckenberg (see [18]) in 1992. They used a simple CA model with periodic borders representing a circular roadway to explain the development of spontaneous congestion forming without an external cause, for example an accident or construction site. They showed that such congestion can form on roads with high-density traffic when cars slow down to avoid colliding with the preceeding vehicle. Resulting from a driver's non-zero reaction time when the preeceding car eventually accelerates, a ripple effect emerges that slowly moves backwards through the stream of succeeding cars.

The Nagel/Schreckenberg model has continously been extended and repurposed. In a recent study, Iwan et al. (see [9]) extend the basic configuration to estimate the environmental impact of unloading bays in city centers. They state that such unloading bays are a simple and effective solution to reduce the congestion resulting from urban freight transport and support that argument with results from CA-based experiments. Their experiments with models of the city centers of Szczecin, Poland and Oslo, Norway show a significant impact of the integration of unloading bays on reducing negative environmental effects of urban freight transport: In time periods with a high ratio of freight vehicles - typically in the mornings - pollutants can be reduced by $15-18$ percent by providing adequate unloading bays.

In another road traffic-related application, Wu et al. (see [29]) examine a number of warning systems aimed at helping drivers to decide on whether to stop or go when a green traffic light turns to red right in front of them. They construct a set of scenarios regarding combinations of warning measures and devise a CA-based model to evaluate them. They find that a combination of pavement markings and flashing yellow auxilliary lights consistently provide the lowest probability of rear end crashes.

In addition to examining road traffic phenomena, a tradition exists of examining ship traffic in harbors and waterways using CA simulation models. Liu, Zhou, and Wang (see [13]) start out with a rather basic simulation model of the traffic flow in single-lane waterways, with Feng (see [4]) adding the representation of length and velocity of different ship types, as well as multi-lane and bottlenecked waterways. Qi, Zheng, and Gang (see [20]) add a more complex system of spatial representation and find that their model maps the ship traffic in the Singapore Strait satisfactionally.

\subsection{Simulation of Additive Manufacturing Processes}

Additive manufacturing (AM) is a set of relatively new technologies to individually fabricate complex parts without expensive individual tooling or set-up cost. One method of AM is selective laser melting, where thin layers of metal powder are one by one melted by a focused laser beam. In this area, understanding and predicting microstructures of manufactured materials and their behavior has seen significant research focus. Both two-dimensional and three-dimensional CAs are applied here, the former to simulate individual layers, the latter to examine the whole structure.

Zinoviev et al. (see [30]) develop a twodimensional, CA-based model that provides a realistic prediction of the evolution of layers of grain structure produced by multiple beam passes during laser melting. Their simulation model yields typical phenomena found in real-world data, for example grain growth competition, through-the-thickness grain growth, and texture formation. They note that, to fully explore the characteristics of the built-up of grain structure, a 3D model would have to be developed.

Rai, Markl, and Körner (see [22]) start out on another approach to simulate grain structure evolution based on a combined 2D CA-Lattice Boltzman model. Their complex model represents a set of major aspects of AM, including random powder layer generation, electron beam energy absorption, evaporation, capillarity and wetting, meltpool dynamics, temperature evolution, and grain solidification. Akram et al. (see [1]) specifically examine the solidification texture of melted metal powder. Understanding that phenomenon can provide a pathway to better understand and control these features, leading to a stream-lined process with better results.

Taking the step from examining individual layers to modeling the whole AM process, Zinovieva, Zinoviev, and Ploshikhin (see [31]) develop a 3D model com- 
bining CA with the finite difference method: The CA model is used to simulate the evolution of grain structure in the solidification processes, while the coupled finite difference method describes the thermal processes in the course of the laser passing through the powder. They specifially examine the creation of titanium parts and find the simulation results reproducing the main features of the observed process. They conclude that their model can be applied to understand the variability in the AM process and the resulting titanium parts.

\subsection{Simulation of Urban Growth and Development}

During the last few years, the examination of urban growth and development using simulation models has seen strong research attention. Two-dimensional CA are obvious candidates for the spatially explicit simulation of land-use and land-cover changes.

Based on urban growth models developed by Clarke, Hoppen, and Gaydos (see [3]) in the 1990s, Sakieh et al. (see [23]) examine cause-effect relationships of urban growth using the example of Karaj, an Iranian city that experienced a substantial increase in urban sprawl during the last three decades. Following on model validation using historical data, they predict urban growth scenarios up to the year 2040, including extensive growth as well as more compact development patterns. They find that, should urban development not be reigned in, the urban land-use in Karaj will grow drastically in the cause of the next two decades. In a similar study, Liao et al. (see [11]) demonstrate the applicability of CA to predict urban growth using the city of Xiamen, China as an example. With good results, Feng, Liu, and Batty (see [5]) apply a more complex CA model to hindcast growth patterns in the Shanghai Qingpu-Songjiang area of China.

To model extreme weather events caused by climate change, urban development models can be augmented by CA-based models of fluvial and pluvial flooding, as described by Guidolin et al. (see [7]).

Moving from conducting individual case studies to preparing meta-analyses and literature reviews, Berberoglu, Akin, and Clarke (see [2]) compare a similar CA model to a number of other methods aimed to assess and predict urban growth based on a model of the city of Adana, Turkey. They compare the simulation results to satellite and aerial images and find that the CA model yields the most exact results. Some weaknesses remain: namely in the quantitative aspect and the in- ability to include the driving forces of urban growth in the model. They recommend to overcome these weaknesses by combining CA with other quantitative approaches, such as Markov chains and frequency ratio models.

\section{Conclusion}

This paper presented an overview on Cellular Automata-based modeling and simulation, describing neighhorhood concepts, border types, and transition functions. It shared some current applications of the paradigm in the areas of the simulation of transportation networks, additive manufacturing, as well as urban growth and development.

Cellular Automata are an elegant, simple, and powerful modeling and simulation technique, naturally employed if the system to be examined consists of a set of uniform entities located in a one- or more-dimensional neighborhood relation to each other. CA-based simulation models have been applied in many research fields for their explanatory power as well as for their high execution performance.

In the sixth decade since their inception by John von Neumann, researchers and practitioners find new applications and gain new knowledge through the exploration and utilization of Cellular Automata. It should be fascinating to watch what the future will bring for them.

\section{References}

[1] Akram J, Chalavadi P, Pal D, Stucker B. Understanding grain evolution in additive manufacturing through modeling. Additive Manufacturing, 21 (2018), pp. 255268.

[2] Berberoglu S, Skin A, Clarke K C. Cellular automata modeling approaches to forecast urban growth for adana, Turkey: A comparative approach. Landscape and Urban Planning, 53 (2016), pp. 11-27.

[3] Clarke K C, Hoppen S, Gaydos L. A self-modifying cellular automaton model of historical urbanization in the San Francisco Bay area. Environment and Planning B: Planning and Design, 24 (1997), pp 247-261.

[4] Feng H. Cellular Automata Ship Traffic Flow Model Considering Integrated Bridge System. International Journal of $u$ - and e-Service, Science and Technology, 6 (6) (2013), pp. 121-132.

[5] Feng Y, Liu Y, Batty M. Modeling urban growth with GIS based cellular automata and least squares SVM 
rules: a case study in Qingpu-Songjiang area of Shanghai, China. Stochastic Environmental Research and Risk Assessment, 30 (2016), pp. 1387-1400.

[6] Gardner M. Mathematical Games - The fantastic combinations of John Conway's new solitaire game "life". Scientific American, 223 (4) (1970), pp. 120-123.

[7] Guidolin M, Chen A S, Ghimire B, Keedwell E C, Djordjevic S, Savic D A. A weighted cellular automata 2D inundation model for rapid flood analysis. Environmental modeling and Software, 84 (2016), pp. 378-394.

[8] Ingalls R. Introduction to simulation. Proc. 40th Winter Simulation Conference, 2008, pp. 17-26.

[9] Iwan S, Kijewska K, Johansen B G, Eidhammer O, Malecki K, Konicki W, Thompson R G. Analysis of the environmental impacts of unloading bays based on cellular automata simulation. Transportation Research Part D: Transport and Environment, 61(A) (2018), pp. 104-117.

[10] Joisten M. Simulation von Fahrplänen für den ÖPNV mittels Zellularautomaten. Köln, Universität zu Köln, Diplomarbeit, 2002.

[11] Liao J, Tang L, Shao G, Su X, Chen, D, Xu T. Incorporation of extended neighborhood mechanisms and its impact on urban land-use cellular automata simulations. Environmental Modelling and Software, 75 (2016), pp. 163-175.

[12] Liebl F. Simulation. 2nd ed. München: Oldenbourg Verlag, 1995.

[13] Liu J, Zhou F, Wang M. Simulation of waterway traffic flow at harbor based on the ship behavior and cellular automata. Proc. 2010 IEEE International Conference on Artificial Intelligence and Computational Intelligence, 2010, pp. 542-546.

[14] Lückemeyer G, Speckenmeyer E. Comparing Applicability of Two Simulation Models in Public Transport Simulation. Proc. 19th Symposion on Simulation Techniques ASIM, 2006.

[15] Macal C M, North M J. Introductory Tutorial on Agentbased Modeling and Simulation. Proc. 2013 Winter Simulation Conference, 2013, pp 362-376.

[16] Maria A. Introduction to modeling and simulation. Proc. 1997 Winter Simulation Conference, 1997, pp. 7-13.

[17] Miksch F, Haim C, Schneckenreither, G. Modelling and Simulation of a SIR-type Epidemic with Cellular Automata and Ordinary Differential Equations. Simulation Notes Europe SNE, 25(1), 2015, pp. 49-54.

[18] Nagel K, Schreckenberg M. A cellular automaton model for freeway traffic. Journal de physique I, 2, 1992, pp. 2221-2229.
[19] Niyat A Y, Moattar M H, Torshiz M N. Color image encryption based on hybrid hyper-chaotic system and cellular automata. Optics and Lasers in Engineering, 90 (2017), pp. 225-237.

[20] Qi L, Zheng Z, Gang L.A cellular automaton model for ship traffic flow in waterways. Physica A: Statistical Mechanics and its Applications, 471(C) (2017), pp. 705-717.

[21] Qin Y, Lu H, Xu Y, Wang H. Saliency Detection via Cellular Automata. Proc. 2015 IEEE Conference on Computer Vision and Pattern Recognition, 2015, pp. 110-119.

[22] Rai A, Markl M, Körner C. A coupled Cellular Automaton-Lattice Boltzmann model for grain structure simulation during additive manufacturing. Computational Materials Science, 124 (2016), pp. 37-48.

[23] Sakieh Y, Amiri B J, Danekar A, Feghhi J, Dezhkam S. Simulating urban expansion and scenario prediction using a cellular automata urban growth model, SLEUTH, through a case study of Karaj City, Iran. Journal of Housing and the Built Environment, 30 (2015), pp. 591-611.

[24] Sarkar P. A Brief History of Cellular Automata. ACM Computing Surveys, 32 (1) (2000), pp. 80-107.

[25] Sun M, Lv H, Zhang Y, Xie G. The Fundamental Primitives with Fault-Tolerance in Quantum-Dot Cellular Automata. Journal of Electronic Testing, 34 (2) (2018), pp. 109-122.

[26] Ullrich O, Lückerath D. An Introduction to DiscreteEvent Modeling and Simulation.Simulation Notes Europe (SNE), 27 (1) (2017), pp. 9-16.

[27] Von Neumann J, Burks A W. Theory of selfreproducing automata. Urbana, University of Illinois Press, 1966.

[28] Weimar J. Simulation with Cellular Automata. 2nd ed., Berlin: Logos Verlag, 2003.

[29] Wu Y, Abdel-Aty M, Ding Y, Jia B, Shi Q, Yan X. Comparison of proposed countermeasures for dilemma zone at signalized intersections based on cellular automata simulations. Accident Analysis and Prevention, 116 (2018), pp. 69-78.

[30] Zinoviev A, Zinovieva O, Ploshikhin V, Romanova V, Balokhonov R. Evolution of grain structure during laser additive manufacturing. Simulation by a cellular automata method. Materials \& Design, 106 (2016), pp. 321-329.

[31] Zinovieva O, Zinoviev A, Ploshikhin, V. Threedimensional modeling of the microstructure evolution during metal additive manufacturing. Computational Materials Science, 141 (2018), pp. 207-220. 